\title{
Décomposition de la phase $\beta$-métastable de l'alliage de titane $\beta$-Cez au cours du chauffage et du recuit
}

\author{
R. SANGUINETTI, M. ZANDONA, A. PIANELLI et E. GAUTIER
}

Laboratoire de Science et Génie de Matériaux Métalliques, URA 159 du CNRS, Ecole des Mines, Parc Saurupt, 54042 Nancy cedex, France

\begin{abstract}
This work carries out the study of the precipitation during continuous heating up to $830^{\circ} \mathrm{C}$ and ageing at this same temperature from $\beta$ meta $+\omega$ aniso or $\beta$ meta $+\alpha$. The heating rates varied between 0.25 and $5^{\circ} \mathrm{C} / \mathrm{s}$. The transfomations were followed by electric resistivity and hardness measurements. Different samples were quenched from different temperatures during the heating range and were then studied by $X$ ray and TEM. The beginning temperatures of precipitations of the $\omega$ and $\alpha$ phases were established, as the continuous heating transformation diagrams. Near $700^{\circ} \mathrm{C}, \mathrm{X}-$ ray quantitative analysis and EAM studies revealed( for quenching and $4.5^{\circ} \mathrm{C} / \mathrm{s}$ cooling rate) that the structure consisted of a maximum content of the $\alpha$ phase, surrounded by $\beta$ zones enriched in $\mathrm{Fe}, \mathrm{Cr}$. For temperatures greater than $700^{\circ} \mathrm{C}$, the content of the $\alpha$ phase diminished and the $\beta$ phase composition was homogenized.
\end{abstract}

\section{INTRODUCTION}

Les alliages de titane riches en éléments $\beta$ gènes comme l'alliage $\beta$-Cez. peuvent après refroidissement présenter une structure $\beta$ métastable à température ambiante ou un mélange de phase $\alpha$ et $\beta$ métastable. Ce travail porte sur l'étude de la décomposition de telles structures métastables au cours du chauffage et du maintien à $830^{\circ} \mathrm{C}$, traitement correspondant à une mise en solution dans le domaine $\alpha+\beta$. Cette décomposition de la phase $\beta$ métastable au cours du chauffage, soit à partir d'une structure $\beta_{\mathrm{m}}+\omega_{\mathrm{ani}}$,soit à partir d'une structure $\beta \mathrm{m}+\alpha$, détermine en partie la morphologie et la distribution des précipités de phase $\alpha$ pendant le traitement de recuit dans le domaine $\alpha+\beta$. Aussi, avons-nous étudié l'effet de la vitesse de chauffage jusqu'à la température de recuit sur la séquence de précipitation au cours du chauffage et du maintien pour le traitement de mise en solution dans le domaine $\alpha+\beta$.

Cette étude a été réalisée en considérant différents "états initiaux" , c'est à dire

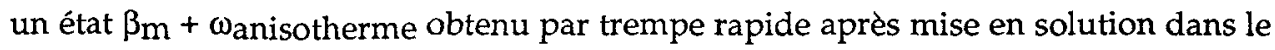
domaine $\beta$, deux états $\beta_{\mathrm{m}}+\alpha$ obtenus pour deux vitesses de refroidissement différentes $\left(4.5\right.$ et $\left.1.5^{\circ} \mathrm{C} / \mathrm{s}\right)$ à partir du domaine de mise en solution $\beta$. 


\section{METHODES EXPERIMENTALES}

L'alliage étudié dénomé $\beta-\mathrm{Cez}$ a la composition nominale suivante: Ti-5\%Al$2 \% \mathrm{Sn}-4 \% \mathrm{Zr}-4 \% \mathrm{Mo}-2 \% \mathrm{Cr}-1 \% \mathrm{Fe}$ (\% en poids). Les traitements thermiques ont été réalisés sur un dilatomètre équipé d'un four à rayonnement qui permet de réaliser des cycles thermiques rapides. Ces cycles thermiques se composent de deux séquences : le traitement de mise en solution dans le domaine $\beta$, suivi d'un refroidissement à différentes vitesses $\left(\mathrm{TR}, 4.5^{\circ} \mathrm{C} / \mathrm{s}, 1.5^{\circ} \mathrm{C} / \mathrm{s}\right)$; et le traitement de mise en solution dans le domaine $\alpha+\beta$, constitué d'une période de chauffage jusqu'à $830^{\circ} \mathrm{C}$, réalisé à différentes vitesses $\left(0.25,0.5,1.25\right.$ et $\left.5.0^{\circ} \mathrm{C} / \mathrm{s}\right)$ puis d'un maintien à cette température de durée variant entre 0 et $60 \mathrm{~min}$. (fig.1)

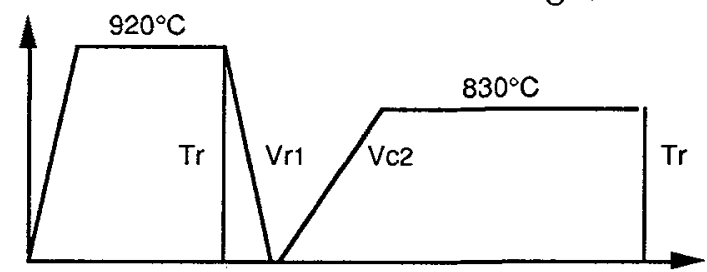

Fig.1 - Traitements thermiques appliqués aux échantillons

Les variations en continu de la longueur et de la résistivité électrique des échantillons en fonction du temps et de la température ont été enregistrées durant le chauffage et le maintien à $830^{\circ} \mathrm{C}$. Les transformations de phases n'ont pu être mises en évidence par dilatométrie. Cependant, des anomalies dans les variations de résistivité ont été observées, pour différentes températures variables avec la vitesse de chauffage. La comparaison à d'autres techniques d'analyse, permet d'associer les variations de cette propriété aux différents changements de phases.

Des échantillons trempés à différentes températures au cours du chauffage, ou différents temps de maintien à $830^{\circ} \mathrm{C}$ ont permis de déterminer les variations de dureté et d'étudier la microstructure et les phases en présence.

La dureté (HV 30) a été déterminée sur des échantillons découpés dans le sens de la longueur, polis mécaniquement afin d'éliminer l'écrouissage préalable. La cinétique de durcissement a donc été obtenue en fonction de la température et du temps de traitement pour différents états initiaux.

Des diffractogrammes $X$ ont également été réalisés en utilisant une anticathode de cobalt, raie $K \alpha 1$ dont la longueur d'onde est égale à $1.7889 \AA$.

Les observations au MET et au MEA ont été faites à partir de lames minces préparées avec l'électrolyte de Blackburn et Williams [1] en utilisant une cellule de jet polissage développée par Bainbridge et Thorne [2].

\section{RESULTATS}

L'étude de la décomposition au chauffage à partir d'une structure obtenue par trempe depuis le domaine $\beta$ a montré que la séquence de précipitation suivante se produit [3] :

$$
\beta_{\mathrm{m}}+\omega_{\mathrm{anis}} \rightarrow \beta \mathrm{m} \rightarrow \beta_{\mathrm{t}}+\omega_{\mathrm{iso}} \rightarrow \alpha+\beta_{\mathrm{r}}+\beta \rightarrow \alpha+\beta_{\mathrm{e}}
$$

où $\beta_{\mathrm{m}}$ est la matrice métastable, $\beta_{\mathrm{t}}$ est la matrice transitoire (présentant des hétérogénéités chimiques), $\beta_{\mathrm{r}}$ est la phase $\beta$ enrichie et $\beta_{\mathrm{e}}$ est la matrice à l'équilibre. 
Les températures de début de précipitation de chaque domaine obtenues par résistivité électrique pour différentes vitesses de chauffage sont présentées figure 2 . En effet, l'état trempé présente un mélange de phase $\beta$ métastable $\left(\beta_{\mathrm{m}}\right)$ et de phase $\omega$ anisotherme, formée au cours du refroidissement. Au cours du chauffage, cette phase $\omega$ anisotherme disparait, puis une phase $\omega^{\prime \prime}$ isotherme" se forme. Ces deux phases ont la même structure et les mêmes paramètres de maille. La phase $\beta$ présente des fluctuations de composition chimique[4], ainsi que l'ont montré nos analyses en M.E.A. Pour des températures plus élevées la phase $\alpha$ précipite et à cette phase $\alpha$, est associé un mélange de phase $\beta$, de composition hétérogène - phase $\beta$ riche correspondant à des zones entourant les précipités $\alpha$ et enrichies en élément $\beta$ gènes (Fe-Cr-Mo) et appauvries en éléments $\alpha$ gènes (Al).

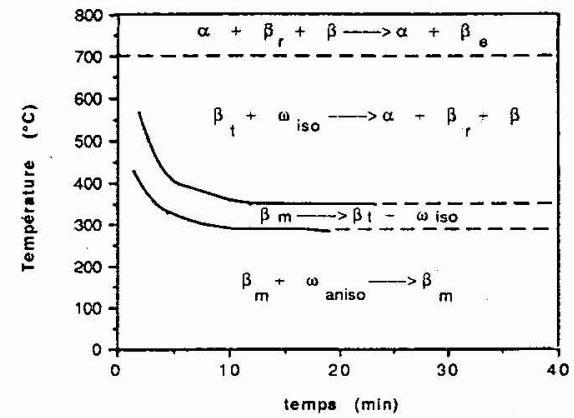

Fig.2- Diagramme TTC pour les échantillons trempés rapidement

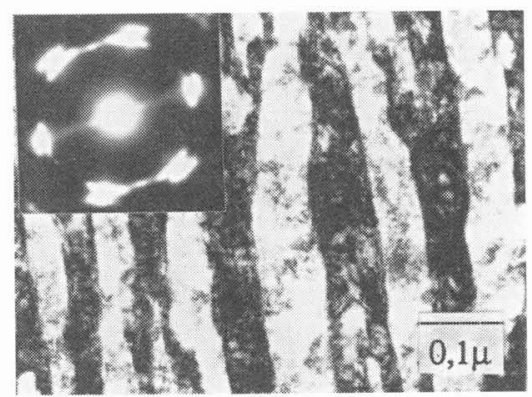

Fig.3- Fond clair et clichés de diffraction (trempe à $680^{\circ} \mathrm{C}$ au cours du chauffage)

La structure après trempe à $680^{\circ} \mathrm{C}$ est présentée figure 3 , et le dédoublement des taches de diffraction des électrons traduit l'hétérogénéité chimique de la phase $\beta$. Un élargissement des raies de diffraction en $R X$ a également été observé. Les analyses EDAX faites sur le même échantillon sont rapportées dans le tableau 1.

\begin{tabular}{|c|c|c|c|}
\hline ELEMENTS & $\begin{array}{c}\text { PHASE } \beta \\
\text { RICHE }\end{array}$ & PHASE $\beta$ & $\begin{array}{c}\text { COMP. } \\
\text { NOMINALE }\end{array}$ \\
\hline $\mathrm{Al}$ & 2.9 & 3.2 & 5 \\
\hline $\mathrm{Mo}$ & 7.4 & 2.2 & 4 \\
\hline $\mathrm{Cr}$ & 5.1 & 2.8 & 2 \\
\hline $\mathrm{Fe}$ & 3.7 & 2 & 1 \\
\hline
\end{tabular}

TABLEAU 1 :

Composition chimique des phases $\beta$ à $680^{\circ} \mathrm{C}$ (\% poids). Analyse EDAX en MET

Ces valeurs montrent pour la phase $\beta$ riche proche des lamelles $\alpha$ un enrichissement en éléments bétagènes ( $\mathrm{Mo}, \mathrm{Cr}$ et $\mathrm{Fe}$ ) et un appauvrissement en $\mathrm{Al}$. De plus, à $700^{\circ} \mathrm{C}$, un taux maximal de phase $\alpha$ est observé toujours associé à cette hétérogénéité de composition chimique, puis pour des températures supérieures (et ce, quelle que soit la vitesse de chauffage), la composition chimique de $\beta$ devient plus homogène et le taux de phase $\alpha$ diminue. Ceci, mis en évidence par $R X$, est visualisé sur les courbes de variations de dureté en fonction du temps (fig. 4). 


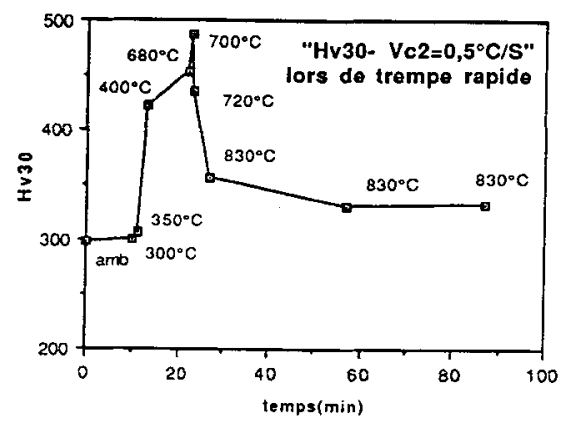

Fig.4- Cinétique de durcissement au chauffage et maintien(Vr1=TR)

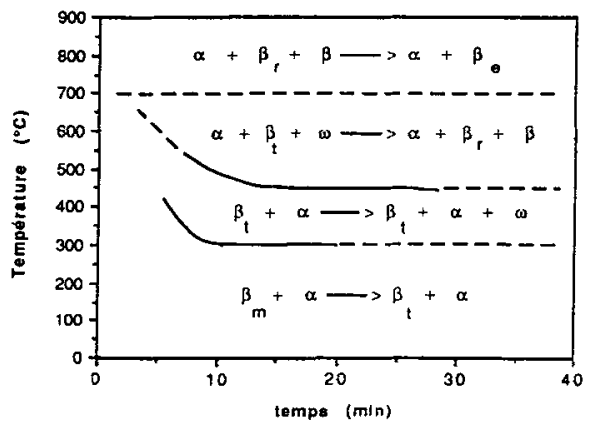

Fig.5- Diagramme TCC pour les échantillons trempés à $4.5^{\circ} \mathrm{C} / \mathrm{s}$

Si nous considérons la décomposition du mélange $\beta_{m}+\alpha$ obtenu à partir d'un refroidissement depuis le domaine $\beta$ à vitesse de $4.5^{\circ} \mathrm{C} / \mathrm{s}$, nous obtenons une séquence de précipitation très voisine de celle obtenue précédemment (figure 5). Les températures de début de précipitation sont modifiées, notamment l'apparition de nouvelle phase $\alpha$ aux vitesses les plus lentes se produit à températures plus élevées) et pour des vitesses de chauffage rapide, la précipitation de la phase $\omega_{i s o}$ peut être évitée. La cinétique de durcissement présente encore une allure voisine (fig. 6).

Enfin, si nous considérons la décomposition d'une phase $\beta_{m}+\alpha$ obtenue depuis le domaine $\beta$ stable par refroidissement à une vitesse de $1.5^{\circ} \mathrm{C} / \mathrm{s}$, nous observons de plus grandes variations de comportement au chauffage. La cinétique de durcissement, figure 7 montre que l'état initial présente une dureté élevée, par rapport aux états initiaux précédents (fig. 4 et fig.6). Cette dureté augmente faiblement jusqu'à la température de $560^{\circ} \mathrm{C}$, puis chute. Les valeurs obtenues à $830^{\circ} \mathrm{C}$ sont les mêmes quelle que soit la structure initiale. Les premières observations en M.E.T. ont montré qu'il n'y a plus précipitation de phase $\omega_{\text {isotherme. }}$

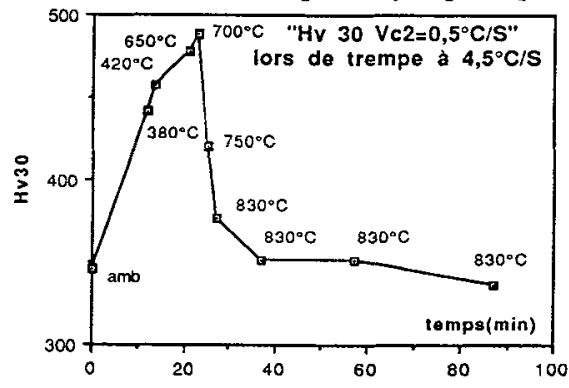

Fig.6- Cinétique de durcissement au chauffage et maintien $\left(\mathrm{Vr} 1=4.5^{\circ} \mathrm{C} / \mathrm{s}\right)$

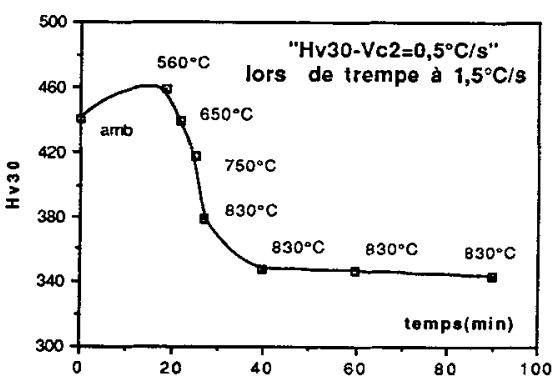

Fig.7-Cinétique de durcissement au chauffage et maintien $\left(\mathrm{Vr} 1=1.5^{\circ} \mathrm{C} / \mathrm{s}\right)$

Enfin, nous pouvons comparer les microstructures obtenues en microscopie optique après mise en solution $\alpha+\beta$ à $830^{\circ} \mathrm{C}$ pendant $30 \mathrm{mn}$ pour les trois états initiaux (fig. 8). Nous obtenons pour l'état initial $\beta_{m}+\omega_{a n i s}$ (trempe à l'eau depuis $\beta$ ) une structrure intragranulaire formée de petits précipités $\alpha$ plus ou moins lamellaire à cette échelle. Sur l'ex-joint de grain $\beta$ a précipité de la phase $\alpha$, très continue, et à son voisinage est observée une zone appauvrie en précipités. 
Pour l'état initial $\beta_{\mathrm{m}}+\alpha$ obtenu par refroidissement à $1.5^{\circ} \mathrm{C} / \mathrm{s}$ la structure est composée de lamelles d'élancement (longueur/épaisseur) très important. Il est délicat de situer l'ex-joint $\beta$. Enfin, la microstructure obtenue pour une structure initiale issue d'un refroidissement depuis $\beta$ de $4.5^{\circ} \mathrm{C} / \mathrm{s}$ est intermédiaire entre les deux structures précédentes.
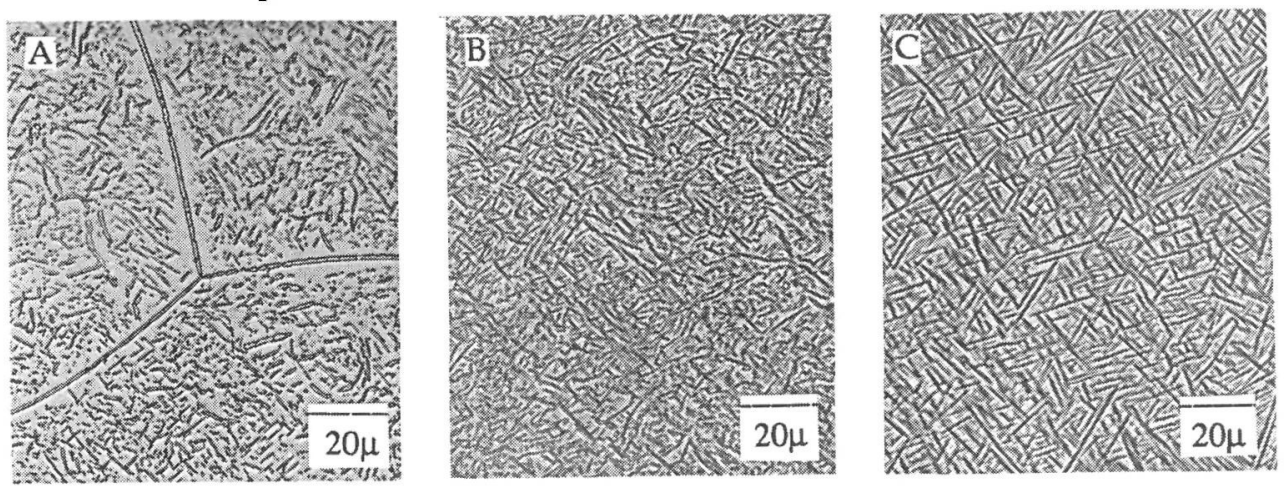

Fig.8- Structures de recuit après mise en solution suivi de refroidissement : a) trempe rapide; b) trempe à $4.5^{\circ} \mathrm{C} / \mathrm{s} ; \mathrm{c}$ ) trempe à $1.5^{\circ} \mathrm{C} / \mathrm{s}$

\section{DISCUSSION}

Les résultats montrent clairement que les transformations de phases, donc la microstructure après recuit (Fig. 8) sont fonction de la vitesse de refroidissement depuis la phase $\beta$ stable et du traitement de chauffage et du maintien à température de recuit dans le domaine $\alpha+\beta$.

La transformation au chauffage de la structure $\beta_{m}+\omega_{\text {anis }}$ ou de la structure $\beta_{m}+\alpha$ présente différentes séquences de transformations [3]. La séquence de précipitation $\omega_{\text {iso }}$ puis $\alpha$ a été rapportée par de nombreux auteurs $[4,5,6]$. La précipitation de $\omega$ a été liée à des fluctuations de la composition chimique de la matrice [3,4] et $\alpha$ germe le plus souvent sur l'interface $\beta / \omega[4,6]$. Ce type d'étude a rarement été réalisé jusqu'à des températures élevées; aussi, l'apparition d'un maxima de phase $\alpha$ à $700^{\circ} \mathrm{C}$ associé à un enrichissement chimique local de la phase $\beta$ n'a pas été rapporté à notre connaissance. Il faut signaler que ce maximum est obtenu par la formation d'un grand nombre de précipités, c'est à dire une vitesse de germination élevée. De plus, la diminution du taux de phase $\alpha$ aux températures supérieures à $700^{\circ} \mathrm{C}$, s'accompagne d'une augmentation de la taille des précipités $\alpha$ (doublement de l'épaisseur de 700 à $830^{\circ} \mathrm{C}$ ) donc une diminution de leur densité. Nous montrons qu'au cours du chauffage jusqu'à $700^{\circ} \mathrm{C}$, le maximum de phase $\alpha$ précipite, et ce pour des vitesses de chauffage allant jusqu'a $5^{\circ} \mathrm{C} / \mathrm{s}$. Pour des températures plus élevées cette phase $\alpha$ se réarrange (coalescence ou autre mécanisme) du fait d'une diffusion plus facile et l'homogénéisation de la phase $\beta$ se produit. 
Si nous considérons les états initiaux différents, une faible vitesse de refroidissement $\left(1.5^{\circ} \mathrm{C} / \mathrm{s}\right)$ conduit, à la température ambiante, à un mélange de phase $\beta$ et de phase $\alpha$ précipités au cours du refroidissement sous forme aciculaire, morphologie liée à la transformation martensitique. Cette structure présente aussi une densité élevée de plaquettes $\alpha$. Pour une telle structure initiale, la phase $\omega_{\text {iso }}$ ne précipite plus et la phase $\alpha$ qui existe à la suite du chauffage, est liée à ces plaquettes formées au refroidissement, et conserve leur structure aciculaire. Un réarrangement des phases (coaelscence ou autre) est toujours observé au cours du maintien à $830^{\circ} \mathrm{C}$.

$\mathrm{Si}$ la dureté de notre alliage à l'issue du traitement de mise en solution à $830^{\circ} \mathrm{C}$, $60 \mathrm{~min}$ est sensiblement identique pour les trois structures initiales, la morphologie est très différente et les propriétés d'usages le seront certainement [7]. Cette morphologie dépend des vitesses de refroidissement depuis le domaine de mise en solution $\beta$, de la vitesse de chauffage et du temps de maintien dans le domaine $\alpha+\beta$. Des morphologies totalement différentes peuvent donc être obtenues. Elles sont liées "au site de formation" de la phase $\alpha$; morphologie lamellaire, mais proche d'une structure équiaxe si la phase $\alpha$ s'est formée à partir d'une phase $\omega_{\text {iso, morphologie }}$ lamellaire/aciculaire, si la phase $\alpha$ s'est formée à partir d'une phase $\alpha$ voire $\alpha^{\prime}$ issue du refroidissement.

L'analyse quantitative de ces microstructures tant pour les taux de phase $\alpha$ que pour la composition chimique des phases ou encore la taille des précipités se poursuit afin d'approfondir les mécanismes de précipitation rencontrés.

\section{REMERCIEMENTS}

Ce travail a été réalisé dans le cadre du G.D.R. "Relations microstructures propriétés des alliages de titane transformés à partir du domaine beta" avec les soutiens de : CNRS, DRET - DGA, AEROSPATIALE, AUBERT et DUVAL, CEZUS, FORTECH DIVISION AIRFORGE PAMIERS, SEP, SNECMA, TURBOMECA.

\section{BIBLIOGRAPHIE}

[1] M.J. Blackburn et J.C. Williams

Trans. AIME, Vol. 239, 1967, 287

[2]- J.E. Bainbridge et $L$. Thorne

Journal Nucl. Mat. Vol.34,1970, 202

[3]- R. Sanguinetti, M. Zandona, A. Pianelli et E. Gautier

3éme EUROMAT, Paris 1993

[4]- G.H. Narayanan et T.F. Archbold

Metallurgical Transactions Vol.1, august 1970, 2281-2290

[5]- F.R. Brotzen, E.L. Harmon et A.R. Troiano

Trans. AIME, Feb.1955, 413-419

[6]- M.H. Campagnac et A. Vassel

Sixth World Conference on Titanium , France 1988, 1619-1624

[7]- L. Mora, C. Quesne, C. Haut, C. Servant et R. Penelle

Rapport GDR Titane, 8 Avril 1990 